\title{
INADEQUACY IN DIAGNOSIS OF BLUNT TRAUMA ABDOMEN - CAN ANAESTHESIOLOGIST BE BAFFLED BY CATASTROPHIC INTRAOPERATIVE FINDINGS?
}

Joyanta Kumar Choudhury1, Dilip Kumar Saloi²

\section{HOW TO CITE THIS ARTICLE:}

Joyanta Kumar Choudhury, Dilip Kumar Saloi. "Inadequacy in Diagnosis of Blunt Trauma Abdomen-Can Anesthesiologist be Baffled by Catastrophic Intraoperative Findings?" Journal of Evolution of Medical and Dental Sciences 2015; Vol. 4, Issue 69, August 27; Page: 12075-12080, DOI: 10.14260/jemds/2015/1739

ABSTRACT: The magnitude of injury inflicted by Blunt trauma abdomen has varied manifestations ranging from minor single-system injury to devastating, multi-system injury .Blunt trauma abdomen alone or in association with polytrauma is a frequent presentation in the emergency department. High index of suspicion and clinical acumen is required during evaluation of blunt abdominal injuries because physical signs and symptoms indicating presence of visceral lesions may poorly correlate with clinical presentation. Diagnostic peritoneal tapping is considered safe initial option with high accuracy but carries possibility of significant false positive and false negative result. Ultrasonography (FAST) has its own limitations; although CT findings can be conclusive it involves time for analysis and the patient should be cooperative. Blunt trauma abdomen may present with life threatening internal haemorrhage due to visceral or vascular injuries prompting emergency surgical intervention at odd hours with limited investigations and resources, Pre anaesthetic optimization by maintenance of adequate tissue oxygenation with optimal blood volume replacement and judicious use of inotropes, determine outcome of such emergency procedures. Careful selection of anaesthetic techniques and drugs particularly the inducing agents can be life-saving. Agility of the attending anesthesiologist to handle unforeseen intra operative critical events plays a pivotal role in overall outcome. We are discussing Anaesthetic management of a 23 year old victim of road traffic accident, who sustained blunt trauma abdomen about $24 \mathrm{hrs}$. Back, was conscious, oriented and ambulatory till the time of shifting to the operating theatre but on exploration sudden gush of blood from a preoperatively undetected $7 \mathrm{cms}$ long tear of the sub diaphragmatic part of IVC, and lacerated liver almost exsanguinated the patient.

KEYWORDS: Blunt trauma abdomen, IVC tear, Liver laceration, Pericardial drain, Thiopentone Sodium.

INTRODUCTION: The spectrum of injury inflicted by Blunt trauma abdomen ranges from minor single-system injury to devastating, multi-system injury. Blunt trauma abdomen alone or in association with poly trauma is a frequent presentation in the emergency department due to various events like road traffic accident, assault, fall etc.

Such injuries demand emergency surgical intervention after possible systemic evaluation and pre anaesthetic optimization, however big vessel injuries may necessitate urgent intervention without adequate evaluation, when careful selection of anaesthetic techniques and drugs along with agility of the anaesthesiologist to handle unforeseen intra operative critical events can be lifesaving.

In spite of availability of rapid transport facilities with trained responder's mortality of patients with intra-abdominal injuries are high.

Survival of patients with big vessel injuries depends upon nature and extent of injuries, pre hospital support, treatment and status of the patient on admission. ${ }^{1}$ 
High degree of suspicion and clinical acumen is required during evaluation of blunt abdominal injuries because the clinical problem being the poor reliability of the physical signs and symptoms that indicate the presence of visceral lesions.,3 and subsequent abdominal distension and presence of significant vascular injuries. ${ }^{4}$ several studies observed inaccuracies in clinical diagnosis by physical examination due to altered levels of consciousness due to alcohol, drugs or neurological deficits. 5,6

Systemic evaluation and diagnostic modalities should go hand in hand; diagnostic peritoneal tapping is considered safe initial diagnostic technique with high accuracy.7,8 it has also significant false positive.7,8 and false negative rate. Ultrasonography (FAST) too has some limitations and although CT findings can be conclusive it involves time in analysis.

Early recognition of extent of vascular injuries is important for formulating resuscitative measures like large volume fluid infusion, massive blood and blood component arrangement and timing of surgery.

We present Anaesthetic management of a victim of road traffic accident who sustained blunt trauma abdomen treated in a district hospital then reported to our emergency department conscious, oriented and ambulatory, Emergency surgery was indicated as Ultrasonography (FAST) detected hemoperitoneum.

He was put up for exploratory laparotomy approximately 20 hours after sustaining the injury, in the interim his clinical status was never alarming apart from some marginal drop of blood pressure responding to fluid resuscitation.

Case History: A 23 years old male victim of road traffic accident referred to the Emergency Department from a district hospital. Patient was initially treated in the district hospital with intravenous fluids, and supplemental oxygen via face mask.

On admission patient was conscious and ambulatory. GCS 15/15, Blood pressure 120/80 mm of $\mathrm{Hg}$, pulse 72 /min /regular, on examination no associated injury could be detected apart from some abrasion over right side of temple and right knee. Airway was patent with normal breath sound and air entry to both the lungs. CVS, CNS examination was normal. Abdomen was tender and on needle tapping there no aspirate.

He was admitted to the hospital and suddenly his BP dropped to $96 / 64 \mathrm{~mm} \mathrm{of} \mathrm{Hg}$, but he responded to resuscitation with 2 liters of crystalloids and supplemental oxygen. He gradually developed abdominal distension, abdomen became tense and tender. Patient was conscious and oriented. Emergency surgery was planned.

Investigations: Computed tomography scan ruled out any head and neck injury. Ultrasonography (FAST) detected hemoperitoneum, X-ray chest was normal. Blood samples were sent for hematological and biochemical investigations. All investigations were within normal limits Hemoglobin was 8.2gm/dl. ECG revealed normal tracing.

Anaesthetic Management: The patient was shifted to emergency operation theatre after obtaining informed and written consent. Crystalloid infusion was started immediately through one 18G IV cannula and three units of whole blood ordered.

Catheterization was done and connected to a bag and urine collection was $200 \mathrm{ml}$. A nasogastric tube was inserted for aspiration of the gastric contents. 
The patient was connected to the multi parameter monitor of the Spacelab anaesthesia work station. His pre induction vitals recorded were $\mathrm{SpO}_{2}$ 96\%, blood pressure $110 / 70 \mathrm{~mm}$ of $\mathrm{Hg}$, pulse rate $128 / \mathrm{min}$ and ECG displayed sinus tachycardia. The patient was administered Inj. Glycopyrrolate $0.2 \mathrm{mg}$, Tramadol hydrochloride $100 \mathrm{mg}$. and Ondansetron $4 \mathrm{mg}$.After premedication patient was preoxygenated with $100 \%$ oxygen for 3 minutes and induced with Thiopentone sodium. ${ }^{9}$

Suxamethonium was used to facilitate intubation; the patient was intubated by the attending anaesthesiologist with a 7.5 sized cuffed endotracheal tube. Vecuronium bromide was chosen for ventilation and muscle relaxation all throughout the surgery. $\mathrm{O}_{2}$ and $\mathrm{N} 2 \mathrm{O}$ were used for maintenance of Anaesthesia. Patient was later connected to a Spacelab Anaesthesia work station.

On exploration peritoneum was filled with blood and clot, while handing a sudden gush of blood of around 4liters blinded entire operative field. Blood pressure dropped to $50 / 30 \mathrm{~mm}$ of $\mathrm{Hg}$ and gradually became non-recordable, pulse became non-palpable, however heart rate recorded 148/min and Sp02 dropped to 83\%, immediately abdomen was packed with hot towels to contain bleeding and resuscitation measures were started in war footing. Isoflurane and Nitrous oxide were withdrawn and patient was ventilated with $100 \%$ oxygen. ${ }^{10}$ Colloid resuscitation, blood transfusion along with noradrenaline infusion was started through 2 available $18 \mathrm{~g}$ IV cannula.

On stabilization a triple lumen central venous catheter was placed in the right subclavian vein and central venous pressure (CVP) was monitored which was $3 \mathrm{cms}$ of $\mathrm{H}_{2} \mathrm{O}$. After successful resuscitation and restoration of haemostasis blood sample was sent for $A B G$ analysis from right radial artery; report suggested normal analysis. Hemoglobin was low at 5.6g/dl after transfusion of three units of blood in succession.

Most astonishing finding was a $7 \mathrm{~cm}$ long longitudinal tear of the inferior vena cava in the sub diaphragmatic region which was bleeding profusely and hepatic laceration through and through in the region right of falciform ligament almost transecting it into two parts. Peripheral pulse became non-palpable and heart rate recorded was 39/min when IVC was finally clamped for repair, surgeon was advised to release the clamp intermittently in order to overcome such catastrophic situation.

During surgery the patient was transfused four units of whole blood, 1litre of colloid and 3litres of crystalloids. Urine output was $150 \mathrm{ml}$ at the end of surgery. Neuromuscular blockade was not reversed as the patient was planned for post-operative ventilatory support in ICU.

Operative Procedure: On exploration peritoneum was filled with blood and clot, while handing a sudden gush of around three and half liters of blood blinded the entire operating field. Estimated loss was around three and half liters, somehow haemostasis was attained by packing the abdomen with towels. It was finally established that apart from the bleeding due to hepatic laceration, blood was gushing out from the 7centimetres long IVC tear.

Pericardial cavity was opened to clamp the supra diaphragmatic IVC to repair its sub diaphragmatic tear, after suction clearance of the pericardial cavity it was closed keeping a drainage tube inside. Non-viable segment of the liver was resected and patent bile ducts ligated. Diaphragm was repaired. Abdomen was inspected for further injuries and closed after putting a drain in the Morrison's pouch.

DISCUSSION: Recognition of severity and extent of internal injuries in a patient of blunt trauma abdomen still remains a challenge; hence the attending anaesthesiologist is unsure of forthcoming critical events during the perioperative and the intraoperative periods while managing such cases in emergency scenario with limited resources of all kinds. 
Blunt trauma abdomen is associated with significant morbidity and mortality in spite of improving mechanisms for early mobilization, recognition, and management. In blunt trauma, the solid organs, like liver, spleen, \& kidneys that cannot give in to the impact by elastic deformation are most prone to injury, managing such eventualities or any other anticipated injuries and its adverse outcomes corroborating with clinical presentations and pre-operative diagnosis are familiar to emergency department anaesthesiologists, however possibility of encountering unmeasured potentially fatal damage should always be in mind.

Detection of extent of intra-abdominal injuries in a patient of blunt trauma abdomen still remains a challenge hence the attending anaesthesiologist is always unsure of forthcoming critical events during the preoperative and the intraoperative periods. . In one autopsy study (Hodgson 2000a) $43 \%$ of abdominal injuries were missed during primary screening in an emergency department. 11

The patient sustained injuries due to high intensity impact of a fast moving truck which caused severe blunt abdominal injuries lacerating and transecting the liver, IVC had a $7 \mathrm{cms}$ long longitudinal tear. The clinical problem is the poor reliability of the physical signs and symptoms that indicate the presence of visceral lesions. ${ }^{2,3}$ both false-positive and false-negative findings bear the risk of severe complications to follow eventually.

The patient reported to the Emergency department four hrs. After the injury with blood pressure of $120 / 80 \mathrm{~mm} \mathrm{Hg}$ which came down to $96 / 64 \mathrm{~mm} \mathrm{Hg}$ during the process of evaluation but stabilized to $110 / 70$ after fluid resuscitation. He was put up for surgery after about seventeen hours after admission due to reluctance of his relatives as apparently he was doing well.

Since the time of injury till the time of surgery he was ambulatory, conscious and oriented. Although peritoneal tapping had negative aspirate, decision for surgery was taken on the basis of USG findings of hemoperitoneum.

Clinical presentation of the patient was not in conformity with the intensity and catastrophic nature of injury and such unanticipated situation demand fair amount of skill on the part of attending anaesthesiologists and synchrony between participating surgeons as well.

Induction of anaesthesia causes dramatic fall of blood pressure; 1,2 Thiopentone sodium $200 \mathrm{mg}$ was administered carefully in titrated doses for induction. Although use of both Thiopentone and Ketamine are conventional in such a situation, use of Ketamine in this patient may have invited such a situation even earlier, as Ketamine causes considerably rise in blood pressure there was possibility of dislodging the haematoma holding the IVC rent or bleeding from the hepatic injuries. Isoflurane was used as the patient had fair amount of haemodynamic stability at the time of induction but it was finally withdrawn.

Adequate volume replacement was done with colloids, crystalloids and blood transfusions. , Noradrenalin infusion was started for maintenance of adequate blood pressure and organ perfusion. Blood and blood components were warmed and transfused rapidly after control of haemorrhage. ${ }^{12}$

The patient attained considerable hemodynamic stability by the end of surgery and he was shifted to ICU. His ICU stay was event free, he was ambulatory and transferred to ward on $7^{\text {th }}$ postoperative day.

CONCLUSION: Blunt trauma abdomen has a wide spectrum of damage potential which can range from minor single system injury to devastating multi-system injury. High index of suspicion and clinical acumen is required during evaluation of blunt abdominal injuries because of poor correlation 


\section{CASE REPORT}

between clinical presentation and the physical signs and symptoms that indicates presence of visceral lesions.

Careful selection of anaesthetic techniques, drugs and judicious use of inotropes, along with agility of the attending anaesthesiologist to handle unforeseen intra operative critical events can be life saving.

\section{ABBREVIATIONS:}

IVC- Inferior vena cava,

ICU- Intensive care unit,

FAST- Focused assessment with sonography for trauma.

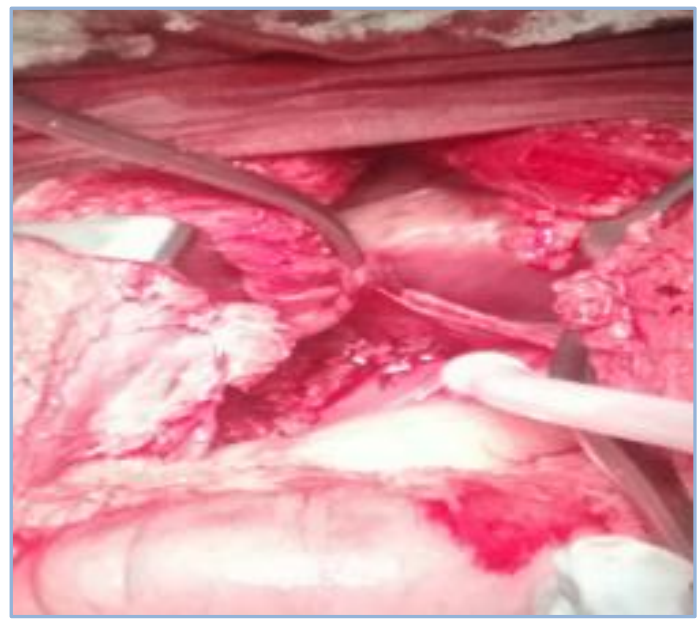

Fig. 1: Exploration of the IVC

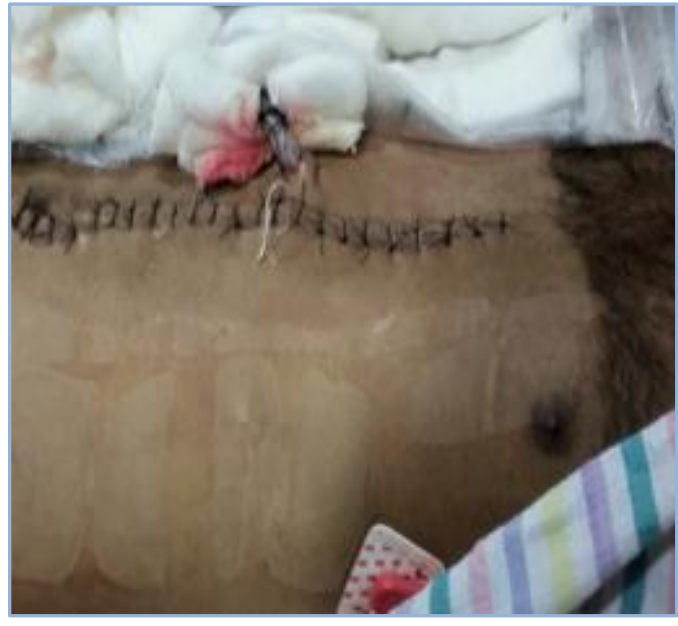

Fig. 2: The Patient with Pericardial Drainage Tube

\section{REFERENCES:}

1. Trinkle JK. Penetrating heart wounds: difficulty in evaluating clinical series. Ann Thorac Surg 1984; 38: 181.

2. Jones TK, Walsh JW, Maull KI. Diagnostic imaging in blunt trauma of the abdomen. Surgery Gynecology and Obstetrics 1983; 157(4):389-98.

3. Prall JA, Nichols JS, Brennan R, Moore EE. Early definitive abdominal evaluation in the triage of unconscious normotensive blunt trauma patients. Journal of Trauma1994; 37(5):792-7.

4. Symbas PN. Cardiothoracic trauma, Philadelphia, W.B. Saunders 1989: 23.

5. Rodriguez A, DuPriest RW Jr., Shatney $\mathrm{CH}$ : Recognition of intra-abdominal injury in blunt trauma victims. A prospective study comparing physical examination with peritoneal lavage. Am Surg 48: 457-459, 1982.

6. Schurink GW, Bode PJ, van Luijt PA, et al: The value of physical examination in the diagnosis of patients with blunt abdominal trauma: a retrospective study. Injury 28: 261-265, 1997.

7. Amoroso TA. Evaluation of the patient with blunt abdominal trauma: an evidence-based approach. Emergency Medicine Clinics of North America 1999; 17(1):63-75.

8. Hodgson NF, Stewart TC, Girotti MJ. Open or closed diagnostic peritoneal lavage for abdominal trauma? Journal of Trauma 2000; 6(48):1091-5. 


\section{CASE REPORT}

9. Toner CC, Stamford J. General Anesthetics as neuroprotective agents. In: Bailliere Tidall, editor. Bailliere's Clinical Anaesthesiology. International pratice and research. UK, Saunders, 1996, Vol 10/ No. 3: 515-33.

10. Anaesthesia for Trauma, Johan K. Stene, Cristopher M. Gande, Anaesthesia 4thEd. Chirchill Livingston 1994: 2157-2173.

11. Hodgson NF, Stewart TC, Girotti MJ. Autopsies and death certification in deaths due to blunt trauma: what are we missing?. Canadian Journal of Surgery 2000; 43(2):130-6.

12. Karim Brohil: Thoracic trauma, Truama.org 6:6, June 2001: 1-3.

\section{AUTHORS:}

1. Joyanta Kumar Choudhury

2. Dilip Kumar Saloi

\section{PARTICULARS OF CONTRIBUTORS:}

1. Assistant Professor, Department of Anaesthesiology and Critical Care, Gauhati Medical College and Hospital, Guwahati.

2. Assistant Professor, Department of Anaesthesiology and Critical Care, Gauhati Medical College and Hospital, Guwahati.

FINANCIAL OR OTHER COMPETING INTERESTS: None
NAME ADDRESS EMAIL ID OF THE CORRESPONDING AUTHOR:

Dr. Joyanta Kumar Choudhury, 2C Ornate Enclave, August Kranthi Path, Opposite Novodaya Jatiya Vidyalaya, Beltola Bazar Road, P.O. Beltola, Guwahati-781028, Assam.

E-mail: drjkc2008@gmail.com

Date of Submission: 18/08/2015. Date of Peer Review: 19/08/2015. Date of Acceptance: 21/08/2015. Date of Publishing: 26/08/2015. 